\title{
THE NUCLEATION AND GROWTH OF GAS BUBBLES IN A NEWTONIAN FLUID: AN ENERGETIC VARIATIONAL PHASE FIELD APPROACH
}

\author{
AARON NABER, CHUN LIU, AND JAMES J. FENG
}

\begin{abstract}
In this paper, we study the nucleation and growth of gas bubbles in a Newtonian fluid. We employ a general energetic variational formulation with a phase-field method, and compare the analytical and numerical predictions of this new formulation with those of classical models. The new approach allows the study of bubble nucleation, growth and coalescence in a unified framework, and has the capability of modeling complex situations in polymer foaming, with multiple-bubble interaction, interaction with an external flow field, complex non-Newtonian rheology and non-Fickian diffusion.
\end{abstract} foams.

Key words. phase field, bubble nucleation, growth, coalescence, Henry's law, Laplace's law,

1. Introduction. Foamed thermoplastics have been in wide use for decades [23], but the relatively mature technology of polymer foaming is facing new challenges. For insulation foams, the mainstay of "blowing agents" - gases dissolved in the polymer melt which form bubbles later-has been hydrochlorofluorocarbons (HCFC's). However, these chlorine-containing chemicals will be banned in the US in 2010 by the Montreal Protocol for their ozone-depleting effects. There has been an intense effort at finding substitutes. A promising group of candidates that provide most of the benefits of the HCFC's are hydrofluorocarbons (HFC's). However, they are less soluble in polymer melts than HCFC's, and the poor processibility poses a challenge for better foaming formulations and process design. Against this backdrop, it becomes an essential and urgent task to understand the mechanisms governing foaming, including the effects of melt viscoelasticity, blowing agent solubility and interfacial tension.

2000 Mathematics Subject Classification. Primary 76T99; Secondary 76R50, 80A99

JJF and CL acknowledge support from The Petroleum Research Fund administered by the American Chemical Society. JJF acknowledges additional support from the NSERC, the Canada Research Chair Program, the Canada Foundation for Innovation and the NSFC (No. 50390095 and No. 20674051). CL acknowledges additional support from the NSF (DMS0405850). 
Foaming is a dauntingly complex process consisting of three main stages. The first is nucleation, where gas in the gas/liquid mixture diffuses into concentrated gas clumps and begins to form bubbles. The second is bubble growth, where bubbles grow by feeding on the dissolved gas in the liquid or even on neighboring smaller bubbles. The third stage is coarsening, where the remaining bubbles coalesce and combine into larger bubbles. In a typical foaming operation, the goal is to produce numerous, uniform-size bubbles. Thus, nucleation in stage one is often promoted by adding nucleating agents, while stage three is suppressed by increasing the extensional viscosity of the melt.

The foaming process is thus dominated by the dynamics of the gas-liquid interface. Conventionally, this interface is treated as an infinitely thin phase boundary across which physical properties change discontinuously. In this sharp-interface framework, the three stages of foaming have to be treated separately, with the end of one stage serving as the initial condition for the next. Nucleation is modeled at best phenomenologically, with much uncertainty regarding the proper initial conditions for the growth stage [15]. Considerable work has been done on bubble growth, albeit limited to the growth of a single bubble in an infinite sea of melt or in a shell meant to simulate the effects of nearby bubbles [46, 7]. Coarsening is often ignored completely, since bubble coalescence involves a topological change of the interfaces that cannot be readily handled by a sharp-interface model. This strategy has proved inadequate, however, for the complexity of the real process. For instance, the single-bubble models predict that the melt viscosity and elasticity should suppress bubble growth [15]. Yet experiments have shown that increasing the melt strength in fact promotes foam expansion, thus producing lighter and better products [44]. This counter-intuitive observation is believed to result from prevention of bubble coalescence and burst; if the melt has low viscosity or elasticity, the film separating neighboring bubbles may become unstable and rupture, a most undesirable effect in foaming. To account for these mechanisms, one must undertake a full-field fluid mechanical analysis of foaming as a highly dynamic two-phase flow.

Toward this end, we propose a diffuse-interface model for foaming that greatly simplifies the treatment of the interface $[31,16]$. In our model, the interface is a layer of minute thickness across which fluid properties change steeply but smoothly. The structure of the interface is determined by the partial mixing between the gas and the melt. Rooted in the microscopic physics of real interfaces, the diffuse-interface theory has distinct advantages for modeling polymer foaming. First, the diffuse-interface method is based on the general principles of mixing and de-mixing of fluid species, 
which underlie critical processes in thermoplastic foaming such as bubble nucleation, interaction and coalescence. Unlike previous models for foaming, it makes no ad hoc assumptions on the shape, size distribution or the mode of interaction among the bubbles. These features will emerge as consequences of the underlying principles. Such a model, based on the physics as opposed to the process, is able to account for the entire process of foaming in a consistent way, from initial nucleation to bubble growth and possibly coalescence, while allowing different bubble shapes and multiple bubbles in a non-homogeneous external flow field.

Second, the diffuse-interface model treats the motion and morphological changes of interfaces much more easily than a sharp-interface description. The flow in the bulk melt is naturally represented in an Eulerian framework while the moving interface requires a Lagrangian description. Reconciling these two viewpoints poses a theoretical and numerical problem in traditional Computational Fluid Dynamics [35]. In our model, we introduce a phase-field variable $\phi$ which varies from +1 in one bulk phase to -1 in the other, and write the governing equations uniformly over the entire domain. The interfaces are simply contours of $\phi=0$, which evolve naturally. This allows us to simulate the motion and interaction of multiple bubbles. Events such as rupture and reconnection of the interfaces, troublesome or even disastrous for the sharp-interface treatment, are simulated with relative ease.

Additional advantages of our model stem from its energy-based formalism. For instance, this ensures an energy law for the mathematical system that plays a central role in the existence and numerical convergence of the solution [31, 34]. Moreover, the variational framework facilitates incorporating complex rheology via a free energy that characterizes the microstructural conformation. Thus, we have chosen the diffuseinterface model over more familiar sharp-interface ones [3, 25] in treating interfacial problems involving complex fluids $[31,49,32]$. In implementation, the diffuse-interface phase-field model is related to other fixed-grid methods such as the volume-of-fluid method [29, 30], the front-tracking method $[20,21,19,8]$ and the level set method $[38,36,18,37,6]$.

The objective of this paper is to demonstrate the applicability of the diffuseinterface model to the nucleation and growth of bubbles. From the classical viewpoint, this problem is governed by diffusion of gas from the liquid into the bubble, with the concentration on the bubble surface determined by the empirical Henry's law. The gas transport into the bubble determines the bubble pressure, which affects the diffusion via Henry's law on the one hand, and controls bubble expansion on the other. Thus, our first task is to show how the familiar concepts of interfacial tension, Fickian 
diffusion and Henry's law can be accounted for in the new theoretical framework. Then, we will quantitatively compare the predictions of our model for the growth of a single bubble with previous results. These two steps will establish the validity of the model in this type of problems. Finally, we present preliminary results on the nucleation and coalescence of a large number of bubbles under conditions resembling micro-cellular foaming. The melt rheology is treated as Newtonian; this and a host of other practical factors will be added in future studies directed more at the physics of the problem than the validity of the methodology.

The rest of the paper is arranged as follows. The theoretical model and numerical methodology will be given in the next two sections, followed by a discussion of the correspondence between the phase-field model and classical models for the foaming process. Section 5 validates the phase-field model for a simple case of bubble growth. Finally, detailed simulation results are discussed in Section 6.

2. Energetic variational formulation. For simplicity, we will constrain ourselves in this paper to the limiting case of incompressibility, $\nabla \cdot u=0$. Moreover, we assume that the density $\rho=\rho_{0}$ is piecewise constant. This is a special case of the conservation of mass equation (except on the interface):

$$
\rho_{t}+\nabla \cdot(\rho u)=0
$$

To describe the flow of two immiscible fluids, say, a gas and a liquid, we introduce an "indicator" function $\phi(x, t)$, where $\{x: \phi(x, t)=1\}$ is the domain occupied by the gas and $\{x: \phi(x, t)=-1\}$ is the region of the liquid. It is clear that the interface between the two materials will be represented by $\Gamma=\{x: \phi(x, t)=0\}$. One can think of $(1 \pm \phi) / 2$ as the volume fractions for the gas and liquid phases. In particular, $\frac{1+\phi}{2}$ will be the gas concentration in the domain. If they are absolutely immiscible, then $\phi$ will be governed by the following transport equation: $\phi_{t}+u \cdot \nabla \phi=0$. The immiscibility is reflected by the fact that a fluid particle is made entirely of one of the two phases, and it does not change type during the flow. The only dynamics is the transport by the flow velocity field.

Remark. If we choose the effective density $\rho$ to be a function of $\phi$, that is $\rho=\rho(\phi)$, Liouville's theorem states that $\rho$ will satisfy the same transport equation as $\phi$ :

$$
\rho_{t}+u \cdot \nabla \rho=0
$$

But this is the same as the conservation of mass equation in the incompressible case. Hence in incompressible, absolutely immiscible fluids, the volume average will be the same as the mass average. 
Here we will employ a slightly different approach by relaxing the condition of absolute immiscibility. Now we can introduce molecular interaction between the species via various mixing and interaction energies that can be derived from the statistical average of the molecules. An example is the Flory-Huggins potential. Here we use the following Ginsburg-Landau type of phenomenological model:

$$
W_{\text {mix }}(\phi)=\lambda \int_{\Omega} \frac{1}{2}|\nabla \phi|^{2}+\frac{1}{4 \epsilon^{2}}\left(1-\phi^{2}\right)^{2} d x .
$$

In this energy, the first term is responsible for the mixing of the two materials. It penalizes the segregation of the two, and thus represents an attractive or "philic" tendency between the species. The second term, on the other hand, penalizes mixing and represents a repulsive or "phobic" tendency between them. For real materials, of course, the interaction between the two species is determined by their chemical properties. The constant $\frac{1}{\epsilon^{2}}$ represents the competition between the two opposite mechanisms. When $\epsilon$ is small, the dynamics will approach that of the classical sharp interface (immiscible case). In fact, as $\epsilon$ approaches zero, $\epsilon W_{\text {mix }}(\phi)$ will tend to the area of the interface $\Gamma$. And then $\epsilon$ can be regarded as the width of the transition layer around the interface. When $\epsilon$ is large, the first term will be dominant and so will be the diffusion mechanism. The model will then correspond to the diffusion models for classical miscible materials, in the sense that the dynamics is determined by the inhomogeneity of $\phi^{1}$. The transition layer with width $\epsilon$ not only serves as a device for regularizing the interface in our model, but also gives a way to model the real mixing between the species, especially when the bubble size is very small.

Remark. When $\phi$ is taken to be the volume fraction, the Flory-Huggins potential also has two terms for mixing and separation, the two competing mechanism:

$$
W_{\mathrm{FH}}=\int_{\Omega}\left[c_{1} \phi \ln \phi+c_{2}(1-\phi) \ln (1-\phi)+\chi \phi(1-\phi)\right] d x .
$$

The difference is that the "philic" mechanism will be due to the consideration of thermal effects, rather than the macroscopic spatial inhomogeneity of the order parameter $\phi$.

With this mixing energy, when we take into account the fact that $\phi(x, t)$ is transported by the fluid velocity field, then it will also induce an extra stress in the momentum equation. After taking the variation with respect to the flow map $x(X, t)$, the induced elastic stress will appear in a weak form such as:

$$
\int_{0}^{T} \int_{\Omega}(\nabla \phi \otimes \nabla \phi, \nabla y) d x d t
$$

\footnotetext{
${ }^{1}$ The classical diffusion equation $\phi_{t}=\gamma \Delta \phi$ possesses the basic energy law $\frac{d}{d t} \frac{\gamma}{2} \int_{\Omega}|\nabla \phi|^{2} d x=$ $-\int_{\Omega}\left|\phi_{t}\right|^{2} d x$. Energetically, the dynamics is totally driven by the inhomogeneity of $\phi$ in the domain.
} 
It is known [31,35] that as $\epsilon$ approaches zero, this stress will reduce to the surface tension on the interface and we recover the boundary condition on a sharp interface (Laplace's law):

$$
[\tau] \cdot n=-m H n,
$$

where the surface tension $m$ is proportional to the limit of $\lambda / \epsilon$, and $n$ is the normal of the interface. The momentum equation is now written as:

$$
\rho\left(u_{t}+(u \cdot \nabla) u\right)+\nabla p-\nabla \cdot(\nu D(u))+\lambda \nabla \cdot(\nabla \phi \otimes \nabla \phi)=g(x),
$$

where the total stress $\tau=-p I+\nu D(u)-\lambda \nabla \phi \otimes \nabla \phi, \nu$ being the viscosity and $D(u)=\left[\nabla u+(\nabla u)^{\mathrm{T}}\right] / 2$ being the stretching tensor, $g(x)$ is an external body force. The pressure $p$ is the combination of a hydrostatic part and an osmotic (elastic) part. The hydrostatic pressure refers to the pressure unattributable to motion and deformation ("static"), not merely due to gravity. In the present case the hydrostatic part is due to the constraint of incompressibility. In compressible cases, it will be due to the internal energy and depend on the density and the temperature.

The above formalism for the mixture of two fluids assumes incompressibility and equal density and equal viscosity between the components. It has also been extended to allow unequal density and viscosity [35]. More recent generalizations have dealt with inhomogeneous surface tension in the form of Marangoni-Bernard convection $[39,24,14,9,33]$ and complex rheology in mixtures of complex fluids $[32,49,50$, $51,52,53]$. In particular, the latter allows for elastic Marangoni stresses that Rey [41] has discussed in the sharp interface framework. From a modeling point of view, incorporating compressibility will not introduce conceptual difficulties.

For a phase function $\phi$, the Cahn-Hillard equation follows the gradient flow (fastest decent) dynamics in certain specific functional space (the dual space of the functional space which includes those functions that are square integrable, and their gradient are also square integrable).

$$
\phi_{t}=\nabla \cdot\left(\frac{\gamma}{\lambda} \nabla \frac{\delta W_{\mathrm{mix}}}{\delta \phi}\right)=-\gamma \Delta(\Delta \phi-f(\phi)) .
$$

Here $\frac{\delta W_{\text {mix }}}{\delta \phi}$ represents the variation of the energy $W_{\text {mix }}$ with respect to $\phi$, and $f(\phi)=$ $F^{\prime}(\phi)$, where the bulk energy $F=\frac{1}{4 \epsilon^{2}}\left(1-\phi^{2}\right)^{2}$ is the second term in the GinsburgLandau energy. The constant $\gamma$ here scales with the reciprocal of the elastic relaxation time of the system.

The solution of (2.8) satisfies the following energy law:

$$
\frac{d}{d t} \int_{\Omega} \frac{1}{2}|\nabla \phi|^{2}+F(\phi) d x=-\int_{\Omega} \gamma\left|\nabla \frac{\delta W_{\text {mix }}}{\delta \phi}\right|^{2} d x=-\int_{\Omega} \gamma|\nabla(\Delta \phi-f(\phi))|^{2} d x .
$$


THE NUCLEATION AND GROWTH OF GAS BUBBLES IN A NEWTONIAN FLUID

In the Cahn-Hillard case, the amount of each species inside $\Omega$ is conserved for all times [5]. It was shown that as $\epsilon$ approaches zero, the dynamics of the Cahn-Hillard equation (2.8) will converge to the dynamics of a Hele-Shaw type flow [1]. This kind of model has been applied previously in the study of spinodal decomposition [10, 12].

Remark. The choice of the Cahn-Hillard dynamics is in order to preserve the total integral of $\phi$. Also, from an energetic point of view (as shown from the above energy law), the difference between this dynamics and the other ones, such as the AllenCahn dynamics $[31,50,48]$, is on the dissipation mechanism, the right hand side of the energy law. Finally, in this case, the chemical potential of the dynamics (2.8) will be propotional to $\frac{\delta W_{\text {mix }}}{\delta \phi}=\lambda(\Delta \phi-f(\phi))$. This chemical potential also provides the nonlocal interactions between the bubbles. As $\epsilon$ approaches zero, it reduces, subject to a coefficient, to the curvature of the bubbles [31]. Hence Cahn-Hillard provides the mechanism of the growth of larger bubbles at the expense of smaller ones - Oswald ripening effect.

With the transport of the fluid, the phase field equation will take the form:

$$
\phi_{t}+u \cdot \phi=-\gamma \Delta\left(\Delta \phi-\frac{1}{\epsilon^{2}}\left(\phi^{2}-1\right) \phi\right) .
$$

Clearly, as $\gamma \rightarrow 0$ (with the elastic relaxation time being much longer than the flow time), the phase field equation will approach the transport equation $\phi_{t}+u \cdot \nabla \phi=0$. This is exactly the kinematic boundary condition of the free interface in the classical immiscible sharp interface models. On the other hand, if we let $\epsilon$ be a large number, the dynamics of $\phi$ will be dominated by diffusion. With flow, the system's energy law becomes:

$$
\frac{d}{d t} \int_{\Omega} \frac{1}{2} \rho|u|^{2}+\frac{\lambda}{2}|\nabla \phi|^{2}+\lambda F(\phi) d x=-\int_{\Omega} \nu|\nabla u|^{2}+\gamma \lambda|\nabla(\Delta \phi-f(\phi))|^{2} d x .
$$

It is important to notice that the energy contributions from the induced stress term and the transport term cancel each other. This is a consequence of the least action principle that is hidden behind the original system.

We shall view our phase field model as intermediate between the classical immiscible sharp interface models and the miscible diffusion models. Compared with those, this model enjoys several advantages:

- It incorporates "phobic" and "philic" mechanisms at the same time, and formulates the concepts of miscibility and solubility in terms of the competition between the two tendencies.

- The model regularizes the interface, and represents the flow of two phases by a single set of governing equations. 
- The formulation can readily incorporate complex fluids with evolving microstructures.

- The model is easy to implement in computations, and handles topological changes in the interfaces with relative ease.

- Although in the simulation in this paper, we concentrate on the "elastic" interaction between the bubbles and set the density to be uniform in the domain, it is relatively easy to incorporate this mechanism either by the Boussinesq approximation or solving an extra transport equation [31, 50]. Hence, although the density ratio is one in the paper, we still refer to the nucleated phase as "gas" for convenience.

Some of the advantages are manifested by the problem to be studied here. Foaming consists of three dynamic processes: bubble nucleation, growth and coarsening. They are inter-related and often take place at the same time. In the traditional sharp-interface framework, each has to be treated separately. For example, nucleation is invariably modeled phenomenologically [15], and coarsening is often ignored altogether since the bubble-bubble coalescence involves a topological change of the interface that cannot be accounted for by the sharp-interface models. The phase field approach allows us to account for all processes from a simple yet profound principle: mixing and separation are governed by the competition between "philic" and "phobic" tendencies between the species contained in the mixing energy. Thus, as the different processes arise from a single set of equations, their activation is not decided on beforehand but is naturally predicted by the model.

3. Numerical Methods. The simulations are based on the coupled CahnHillard and Navier-Stokes equations. All equations are written in dimensionless form, and the dimensionless parameters, denoted with a bar, are discussed in Section 3.1. The simulations done here are almost completely diffusion driven, as opposed to convection driven, with maximum velocities many orders of magnitude below the characteristic length divided by the characteristic time. The small convective effect makes a Galerkin-Fourier Spectral Method particularly suitable. In addition, periodic boundary conditions are always used, which allow us to model the nucleation and growth of many bubbles in an effectively unbounded domain.

Using the divergence free condition $\nabla \cdot u=0$, together with the fact that $u$ is nowhere singular in our problem, we may write $u$ as $u=\nabla \times \mathbf{A}$ for some vector-valued stream-function $\mathbf{A}$. In fact, in two dimensions we simply have $\left.u=<A_{y},-A_{x}\right\rangle$, where $A$ is the $z$ component of $\mathbf{A}$, and $A_{x}, A_{y}$ are its $x$ and $y$ partial derivatives, respectively. Applying this to the vorticity form of the momentum equation, we have the following 
set of equations:

$$
\begin{gathered}
(\triangle A)_{t}+u \cdot \nabla(\triangle A)=R e^{-1} \triangle(\triangle A)-\bar{\lambda}\left(\phi_{x} \triangle \phi_{y}-\phi_{y} \triangle \phi_{x}\right) \\
\phi_{t}+u \cdot \nabla \phi=-\bar{\gamma} \triangle\left(\triangle \phi-\frac{1}{\bar{\epsilon}^{2}}\left(\phi^{2}-1\right) \phi\right) \\
u=<A_{y},-A_{x}>
\end{gathered}
$$

The time discretization is semi-implicit, where the linear terms are done in the Crank-Nicholson style and the nonlinear terms are treated explicitly. The weak convection in the simulations allows the use of such a scheme. This way, each variable has a separate system of linear equations to be solved at each time step, so a conjugate gradient method can be used to solve each system separately. In addition, although the pressure term does not explicitly appear in the equations, we can periodically solve for the pressure by applying a conjugate gradient to the Poisson pressure equation, which, with the additional terms due to the Cahn-Hillard elastic stress, comes out to be

$$
-\triangle p=\nabla \cdot(u \cdot \nabla u+\bar{\lambda} \nabla \cdot(\nabla \phi \otimes \nabla \phi))
$$

The pressure term being solved for is therefore the hydrostatic pressure plus the gradient term that arises due to the original variational principle [31].

Currently, our code is limited to two dimensions by the problem size. A critical element in extending to three dimensions will be an optimized meshing scheme that maximizes resolution near interfaces but coarsens the grid away from them. Two strategies seem promising at present. One is an adaptive meshing technique that dynamically adjusts an Eulerian grid [54], and the other is a moving mesh scheme that incorporates Lagrangian movement of the grid points [17]. Three-dimensional codes using both methods are under development.

3.1. Parameters and Scaling. We denote the characteristic time, length and density by $L, T$ and $\rho$, respectively. Then the dimensionless coefficients $R e, \bar{\lambda}, \bar{\gamma}, \bar{\epsilon}$ are related to the dimensional coefficients by the following relations:

$$
R e=\frac{\rho L^{2}}{T \nu}, \bar{\lambda}=\frac{\lambda T^{2}}{\rho L^{4}}, \bar{\gamma}=\frac{T \gamma}{L^{4}}, \bar{\epsilon}=\frac{\epsilon}{L}
$$

All simulations were run in a square domain with a side length of 100 for one or two bubbles, or 500 for a larger number of bubbles. The grid was uniform, as required 
by the Fourier Spectral Method, with either $N=128$ or $N=256$ grid points along each edge. Adequacy of the spatial resolution was confirmed by mesh refinement.

The diffuse-interface model parameters $\bar{\lambda}, \bar{\gamma}$ and $\bar{\epsilon}$ have to be chosen to match the classical parameters in an experiment or a prior calculation with which we compare our simulation. To illustrate this matching, we consider Venerus and Yala's [46] sharp-interface calculation of the growth of a single bubble in an infinite sea of a Newtonian fluid. In both phase-field and classical calculations, the long-time growth is characterized by a "diffusion-controlled" regime with the scaling $R \propto \sqrt{t}, R$ being the bubble radius [46]. By fitting the slope of the $R \sim \sqrt{t}$ plot to that of the classic solution, we determine our parameter $\bar{\gamma}$. The capillary thickness $\bar{\epsilon}$ and mixing energy density $\bar{\lambda}$ are determined simultaneously by the use of the dimensionless surface tension relation $\bar{m}=\frac{2 \sqrt{2}}{3} \frac{\bar{\lambda}}{\bar{\epsilon}}$ (see [49]) and the matching of the Henry's law constant (see sub-section 4.2). Finally, the Reynolds number $R e=10^{-6}$ is taken directly from the classic solution. This leads to the following parameters, used in all simulations unless otherwise stated:

$$
R e=10^{-6}, \bar{\lambda}=1.74, \bar{\gamma}=17778, \bar{\epsilon}=5.03
$$

4. Classical Physical Laws in the Phase Field Model. In switching from a classical approach to a phase-field model, it is important to understand the roles played in the phase-field model by the physical laws upon which the classical model is based. In particular, we wish to study the Laplace formula for surface tension, Henry's law for the influence of pressure on solubility, and Fick's law for mass diffusion.

4.1. Laplace's Formula. It has been shown [1] that as $\bar{\epsilon}$ tends to zero in the phase-field model, the dynamics governing the mixing of the fluids approach the classical sharp-interface model and, in particular, Laplace's law becomes valid. However, since our simulations involve a finite interfacial thickness, it is important to understand how Laplace's law fits into the model. To this end we ran several simulations.

The first simulation is the relaxation of a circular bubble in a square domain with side length 100. The initial conditions are zero velocity and the phase field

$$
\phi=-0.99 \tanh \left[\frac{\sqrt{(x-50)^{2}+(y-50)^{2}}-20}{5}\right],
$$

which represents a bubble of radius 20 in the center of the domain, and 0.99 is simply a relaxation factor meant to allow the bubble to reach equilibrium more quickly. The above $\phi$ profile is not an energy minimizer, and therefore relaxes toward an equilibrium 


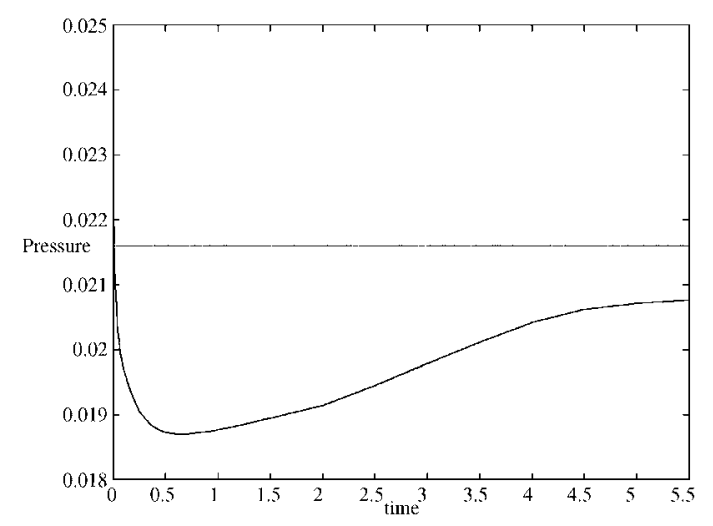

FIG. 4.1. Relaxation of bubble pressure as predicted by our phase-field model. The dotted horizontal line indicates the pressure from Laplace's formula.

state. Figure 4.1 plots the temporal evolution of bubble pressure in this process, where the dotted line is the prediction by Laplace's formula, based on the final bubble radius. Note that the pressure drops steeply at the beginning, and then climbs up toward the Laplace value. In the end, the pressure is within about $5 \%$ of the predicted value.

The second simulation involves the nucleation and growth of many bubbles. A square domain with side length 500 is initially filled with a homogeneous 70/30 mixture of liquid and gas. We impose a random perturbation of the concentration at each grid point so the gas concentration varies between $25 \%$ and $35 \%$. This destabilizes the homogeneous state, and over time the random gas clumps aggregate to nucleate bubbles, which then grow by diffusion and interact with surrounding bubbles. In time an apparent equilibrium point is reached when the bubbles attain steady-state sizes and a circular shape, with no further growth. Rigorously, this is not a true equilibrium but a "quasi-equilibrium" where the bubbles are far enough apart that they do not change much over the time scale of the simulation. At this stage, the bubbles have various sizes and the pressure differences inside and outside the bubbles can be measured. These discrete measurements are compared with the pressure due to the Laplace formula in Figure 4.2. Again, the phase-field model with the coefficients used here, and in particular a relatively large value of $\bar{\epsilon}$, predicts pressure values slightly less than that of the classical model. A more complete description of this simulation and the other phenomena occurring in it can be found in section 6 below.

4.2. Henry's Law. Henry's law states that the amount of gas dissolved in a liquid is proportional to the gas pressure:

$$
p_{g}=k c_{g}
$$




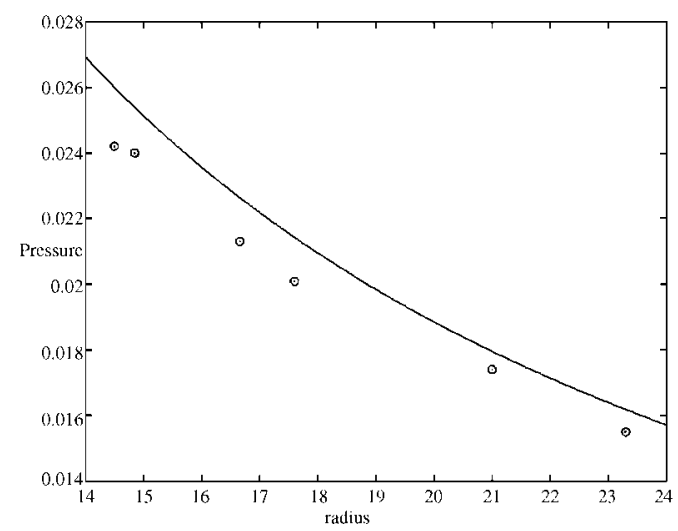

FIG. 4.2. Bubble pressure as a function of bubble radius: comparison between phase-field predictions (data points) and the Laplace law (curve).

where $p_{g}$ is the gas pressure, $c_{g}$ is the equilibrium gas concentration, and $k$ is the Henry's law constant. This linear relationship can be derived from the thermodynamics of solutions in the limit of low solubility of an ideal gas $[26, \S 88]$. Empirically it is found to hold if the pressure is not too high.

In our phase-field model, we do not have, nor need, a Henry's law. Instead we can derive a relationship between the gas concentration and the osmotic pressure that resembles Henry's law. The purpose of making this connection is to set the backdrop against which the model predictions can be related to empirical observations.

Since we have assumed incompressibility for both components, strictly speaking we cannot model Henry's law for an ideal gas. But the mixing energy affords an interesting relationship between gas concentration and the osmotic pressure, which can be considered the counterpart of Henry's law in our case. Let $\phi=1$ for the pure gas phase inside the bubble. Just outside the bubble, the gas-melt mixture assumes a value $\phi=\phi_{0}$ between 0 and -1 (Fig. 4.3). A variation of the mixing energy in Eq. (2.3) gives rise to the following elastic stress tensor (cf. [49]):

$$
\tau=-\left(p_{t}-W_{\text {mix }}\right) I-\lambda \nabla \phi \otimes \nabla \phi,
$$

where $p_{t}$ is the thermodynamic pressure and $W_{\text {mix }}=\lambda\left(|\nabla \phi|^{2} / 2+\left(\phi^{2}-1\right)^{2} / 4 \epsilon^{2}\right)$ is the mixing energy. Inside the bubble, the bulk mixing energy $F=0$ since $\phi=1$. Outside the bubble, $F=F_{0}=\lambda\left(\phi_{0}^{2}-1\right)^{2} / 4 \epsilon^{2}$. Thus, the osmotic pressure across the bubble surface is

$$
p_{o}=F_{0}=\frac{4 \lambda}{\epsilon^{2}} c_{g}^{2}\left(1-c_{g}\right)^{2},
$$

where $c_{g}=(1+\phi) / 2$ is the gas concentration. This relationship between the osmotic pressure and the gas concentration corresponds to Henry's law for ideal gases. The 


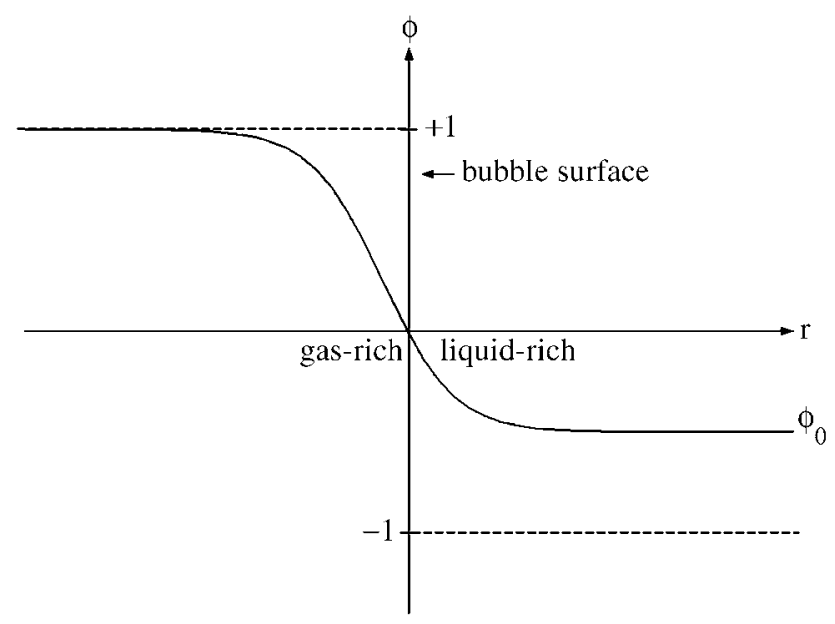

FIG. 4.3. A sketch of the $\phi$ profile across the bubble surface. The osmotic pressure is related to the gas concentration outside the bubble by a relationship akin to Henry's law.

leading-order term being quadratic in $c_{g}$ is due to our choice of the mixing energy $W_{\text {mix }}$. Indeed, if we use the Flory-Huggins energy, the leading term will be linear in the gas concentration.

In sharp-interface models for diffusion-induced bubble growth, Henry's law is used to determine the gas concentration outside the gas bubble, which serves as a boundary condition for the diffusion equation in the melt $[2,15,40]$. In our formalism, such a boundary condition is unnecessary. Instead, we use the correspondence between Eqs. (4.2) and (4.4) as a condition to determine the parameter values $\lambda$ and $\epsilon$. More specifically, the osmotic pressure $p_{o}$ and the gas pressure $p_{g}$, averaged over all concentrations $0 \leq c_{g} \leq 1$, are equated. This provides a means to incorporate the Henry's law constant $k$ into our model.

4.3. Fick's Law. In the foaming literature, the diffusion of the dissolved gas is usually modeled by Fick's law, which is based on the idea that mass diffusion is driven by gradients in composition. Moreover, the diffusivity is usually taken to be a constant. From statistical mechanics, however, equilibrium requires a uniform chemical potential, which does not necessarily imply a uniform composition. Thus, a generalized Fick's law can be postulated whereby the mass flux is proportional to the gradient of a chemical potential $\delta W_{\text {mix }} / \delta \phi$. This immediately leads to the CahnHillard Eq. (2.8). Expanding the right hand side of this equation using the double-well 
bulk energy, we arrive at:

$$
\phi_{t}=\nabla \cdot\left(f^{\prime}(\phi) \nabla \phi\right)-\gamma \Delta \Delta \phi
$$

where $f^{\prime}(\phi)=\left(3 \phi^{2}-1\right) / \epsilon^{2}$. The biharmonic term on the right hand side, due to the gradient energy in $W_{\text {mix }}$, makes the Cahn-Hillard diffusion non-Fickian. The first term resembles Fickian diffusion with a diffusivity of $f^{\prime}$, which can be either positive or negative. The zeros of $f^{\prime}$ define the spinodal lines: $\phi_{\text {spin }}= \pm 1 / \sqrt{3}$. Within these limits, the Cahn-Hillard system exhibits "negative diffusion"; the instability leads to spinodal decomposition. Beyond the limits, normal diffusion prevails. We will revisit spinodal decomposition in sub-section 6.1 with concrete numerical results. The presence of the gradient term in the mixing energy produces a nonlocal diffusion mechanism in space. It provides a growth mechanism for the bubble, through this generalized diffusion, even when the local gas concentration gradient outside of the bubble is temporally zero.

It is well-known that small-molecule substances often follow non-Fickian diffusion in polymer melts $[42,43,4]$. This can be attributed to the fact that the penetrant molecules disturb the polymer chains, whose relaxation produces an additional viscoelastic stress. The phenomenological Cahn-Hillard model, of course, was not built specifically to reflect this process. Nevertheless, it provides us with a way to model non-Fickian diffusion of dissolved gas in polymer melts. In fact, such a non-Fickian term plays an important role in the bubble growth process in our theory. Without gas compressibility and the conventional Henry's law, the gas concentration at the bubble surface is usually not much lower than that in the far field. Thus, the classical process of gas diffusion through the melt toward the bubble is not adequately represented by the Fickian term. The non-Fickian (and nonlocal) character of the Cahn-Hillard diffusion provides the needed mechanism for bubble growth.

5. Validation of Phase-Field Results. In validating the phase-field model it is important to compare its results with those of the classical model when possible. The classical model itself is limited to the simplest situation, the spherically symmetric growth of a single bubble. We therefore start there and show a relation between the results. Then we simulate more complicated situations with two and several dozen bubbles. By comparing the growth of each individual bubble in the complicated case to the single bubble case, we evaluate how good an approximation it is to use the single bubble growth curve to predict the behavior of numerous bubbles, as is done in the classical case [15]. 


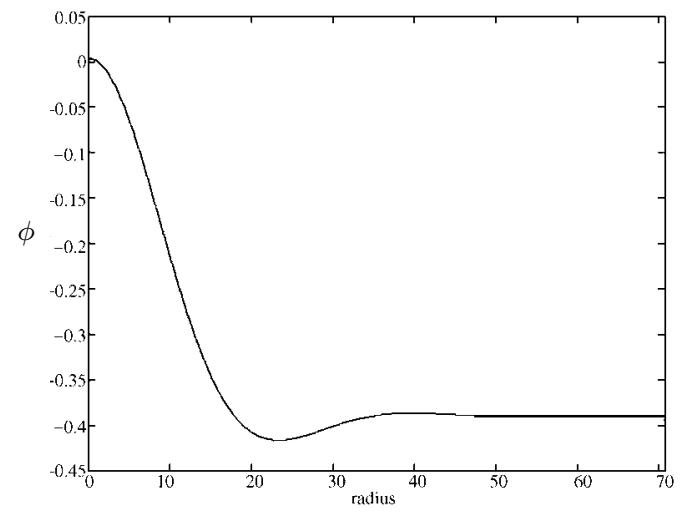

FIG. 5.1. The $\phi$ profile for a pre-existing bubble that is used as the initial condition for our first simulation. The abscissa is the distance from the center of the bubble.

5.1. Single Bubble. We compare two simulations with the results of Venerus and Yala [46], who computed the growth of a bubble in an infinite domain of a Newtonian melt using a classical sharp-interface model. In this work, the process of nucleation is not considered; instead, a bubble with a certain radius and initial properties is used as the initial condition. Similarly, in our first simulation, we disregard the nucleation stage and start with a pre-existing bubble. In the second, we perturb a homogeneous mixture to initiate nucleation at multiple sites, which is then followed by bubble growth.

In the first simulation we start with an initial bubble centered in a square domain. The initial conditions are zero velocity and a radially symmetric $\phi$ profile depicted in Figure 5.1, which is extracted after a small time interval. Note that $\phi>0$ in the center, which is the interior of the bubble. The initial bubble radius $R_{\text {init }}=1$ matches that in [46]. To match the slope of the diffusion-controlled growth curve [curve (a) in Figure 5.2], we use a smaller value for $\bar{\gamma}: \bar{\gamma}=8889$.

The result is shown in Figure 5.2 by curve (c), which exhibits much the same behavior as curve (b) representing the classical solution. The main difference occurs in the early stage of bubble growth, where the phase-field solution attains the longtime asymptotic growth sooner than the classical solution. There are two possible causes for this. First, the combined Fickian and non-Fickian diffusion discussed earlier may contribute to this phenomenon. Second, this may also be due to the lack of compressibility in our phase-field equations. As the gas diffuses into the bubble, its volume remains the same in our model. This produces a lower bubble pressure and a lower gas concentration on the interface. Hence faster gas diffusion into the bubble. The effect of the compressibility will be discussed in detail in a future publication. 


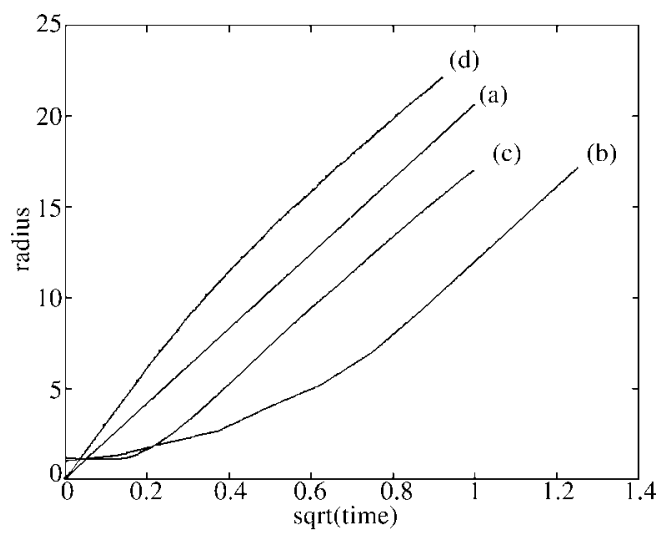

FIG. 5.2. Comparison of single bubble growth between classical and phase-field models. The solid line (a) indicates the diffusion-controlled limit with $R \propto \sqrt{t}$. Curve (b) is the classical result from [46]. Curves (c) and (d) are our results without and with the nucleation stage, respectively.

In our second simulation, we start with zero velocity and a $\phi$ distribution:

$$
\phi=-0.3-0.1 \tanh \left[\frac{\sqrt{(x-50)^{2}+(y-50)^{2}}-16}{4.62}\right] .
$$

Such an initial condition represents not a bubble, but a small gas clump in a 70/30 liquid/gas mixture. As the simulation continues, nucleation occurs and the clump grows into a bubble. Then the bubble expands through diffusion of gas from the outside mixture into the bubble. Once the bubble has formed we measure the growth of the bubble and plot the data in Figure 5.2 as curve (d). The long-time behavior is similar to the first simulation, showing the diffusion-controlled asymptotic growth. But in a short interval of time after nucleation, the slope of the $R \sim \sqrt{t}$ curve is greater than the asymptotic slope. This contrasts curves (b) and (c), which have started from a pre-existing bubble without nucleation. This discrepancy highlights one of the failings of the classical models: its inability to integrate bubble nucleation with growth. Ignoring the former, one has to postulate an initial bubble and prescribe its initial properties. Such initial conditions have been subject to much debate [15, 45]. Those used in $[46,15]$ assume an initial force balance on the bubble surface so the bubble starts with zero acceleration. This may have caused the slow initial growth of the classical result represented by curve (b) in Figure 5.2.

5.2. Multiple Bubbles. Next we have carried out a simulation with two bubbles near each other. The idea is to compare the growth rate of each bubble with the single bubble solution. The simulation was run in a square domain with side length 100. The initial conditions had zero velocity and a 70/30 liquid/gas mixture with two gas clumps having liquid/gas mixture of 60/40 and radius 16 , whose centers are 


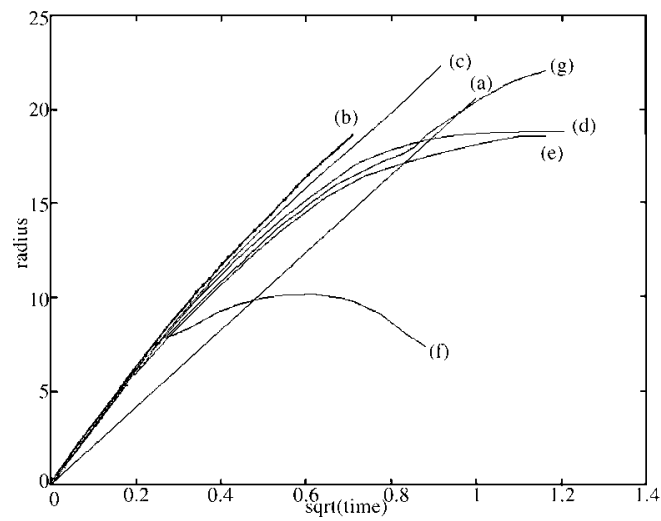

FIG. 5.3. Growth of bubbles in the presence of nearby bubbles. The solid line (a) indicates the diffusion-controlled limit. Curve (b) is the growth of one bubble in a two-bubble pair, and is essentially identical to the single bubble growth curve in Figure 5.2, re-plotted here as (c). Curves $(d, e, f, g)$ represent 4 bubbles in a foam containing dozens of bubbles (cf. Figure 6.1).

located a distance of $50 \sqrt{2}$ apart. The growth of each bubble is shown by curve (b) in Figure 5.3. It is essentially identical to the single bubble case, curve (d) in Figure 5.2. Therefore, as long as the bubbles are sufficiently far apart, as they are in this simulation, they do not interact with each other and their behavior can be predicted by single-bubble simulations.

Now we consider a situation with several dozen bubbles in close proximity so that their interaction stunts the growth of some bubbles. The simulation is described in detail in section 6 . A homogeneous $70 / 30$ liquid/gas mixture is subject to a spatially random perturbation of the concentration. The random gas clumps then serve as seed sites for bubble nucleation. Once the gas clumps become bubbles, we track several of the bubbles during their growth, and compare the results to the single bubble growth curve in Figure 5.3. Curves (d) and (e) follow the single-bubble growth curve up to a certain point, and then they level off since the bubbles begin to interact with their neighbors. The growth curve (f) for the third bubble breaks away from the singlebubble case much earlier; it levels off, and then begins to decrease at a rapid rate. This is because the third bubble has close neighbors with which it interacts strongly from the beginning. It eventually diffuses into the surrounding bubbles. Curve (g) follows the paths of (d) and (e) for a period of time, and then begins a sudden increase. In fact, the bubble represented by $(\mathrm{g})$ grows at the expense of the bubble represented by (f), whereby (f) diffuses into (g) by the growth/shrink process discussed in Section 6.2. From this comparison, we conclude that applying single-bubble dynamics to multiple bubbles is invalid as soon as they start to interact. 
6. Numerical Results on Foaming. As mentioned before, the foaming process consists of bubble nucleation, growth and coalescence, and to account for all three stages in a self-contained theory is our primary motivation to adopt a phasefield model with diffuse interfaces. The phase-field model used here is built on the dynamics of mixing fluids, with no special attention to the foaming stages. Yet, as we demonstrate below, with a simple initial condition we can reproduce all three stages in the correct order. As in an actual foaming process, diffusion is the dominant physics throughout. But each stage has its unique mechanism that drives the system. For the nucleation stage, it is phase separation via spinodal decomposition or nucleation and growth. In the growth stage, the bubbles are forced to interact through a diffusion process where larger bubbles grow at the expense of smaller ones. In the final stage, of course, the mechanism of importance is the coarsening itself.

We simulate a complete foaming process in a square domain with side length 500. Initially the domain is filled with a homogeneous gas-liquid mixture with $30 \%$ gas. Velocity is zero everywhere. Now we impose a random disturbance of the concentration at all grid points so the gas concentration varies between $25 \%$ and $35 \%$. This inhomogeneity allows gas clumps to randomly form throughout the domain, which become bubbles in time and set off the foaming process. Figure 6.1 illustrates this process using snapshots. The first picture shows the initial state. The next two show the nucleation process, with the gas clumps beginning to collect in the second picture, and the bubbles fully formed in the third. The following two pictures show the bubble growth stage, with smaller bubbles being "eaten" by larger neighbors. The arrows indicate two examples of this process. The final picture shows the beginning of the coarsening stage. The bubbles continue to grow, and begin to coalesce with neighbors once they get too close. In the following, we will analyze each stage in greater detail, sometimes with supplemental results.

6.1. Stage I: Nucleation. The Cahn-Hillard dynamics gives a phenomenological explanation of the phase separation of two mixtures [10,12]. Many models have been proposed for this process [13, 27], and Larson's [28] discussion of spinodal decomposition versus nucleation and growth is particularly relevant to our system. The double-well bulk energy $F(\phi)=\frac{1}{4 \epsilon^{2}}\left(1-\phi^{2}\right)^{2}$ has two minima at $\phi= \pm 1$. Thus, it favors complete separation between the gas and the liquid. The spinodal concentrations can be determined from the inflection points of $F(\phi): \phi_{\text {spin }}= \pm 1 / \sqrt{3}$, which correspond to a gas concentration of $21.1 \%$ and $78.9 \%$. Therefore, if the initial concentration falls between these two numbers, the system undergoes spinodal decomposition and instantaneously separates into a bi-continuous network. This will in 

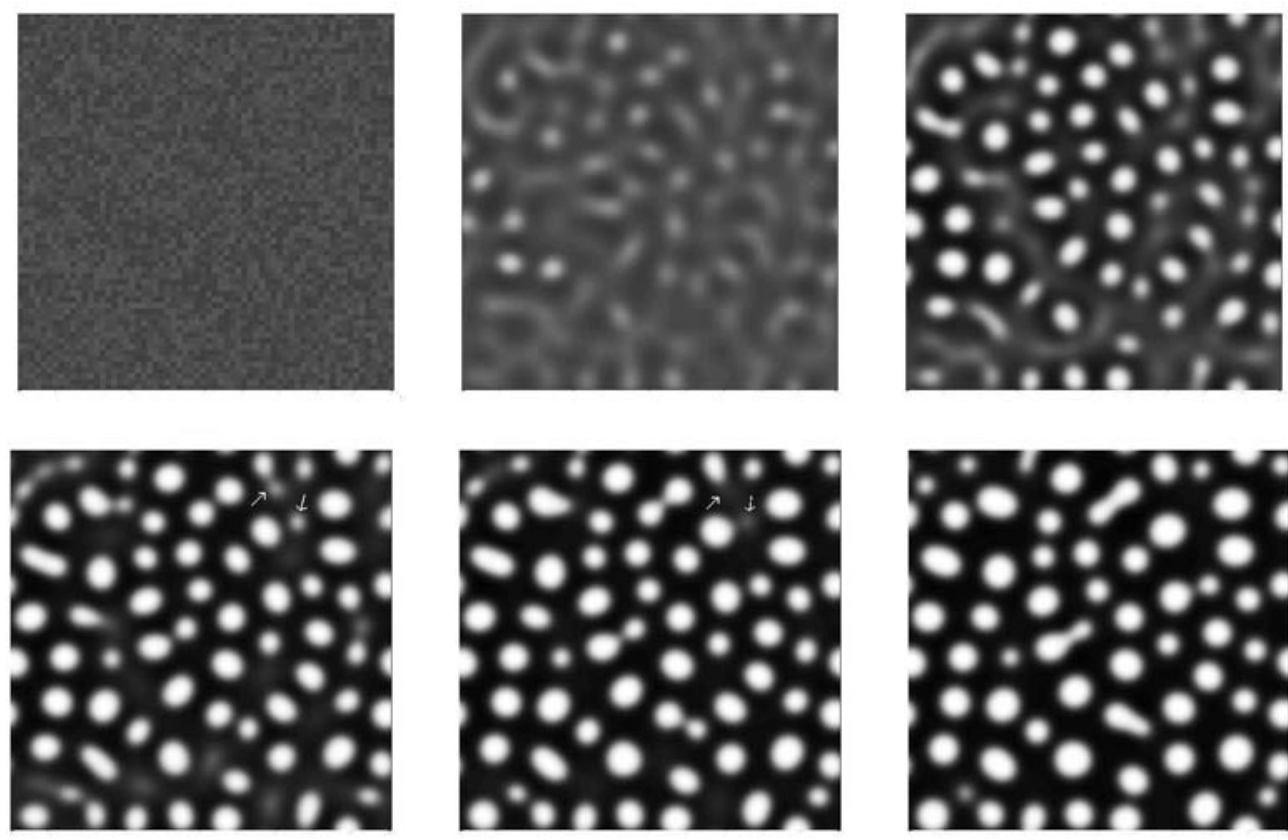

FIG. 6.1. Snapshots of the foaming process at times $t=0,0.66,1.2,1.5,1.75$ and 2.1, showing the nucleation, bubble growth and coarsening stages. Arrows in slides 4 and 5 point out examples of small bubbles being absorbed by larger neighbors.

time break down to a drop-in-matrix morphology unless the two species are perfectly balanced at $50 \%$ each. If the gas concentration is below $21.1 \%$ or above $78.9 \%$, the system is metastable and separation may only occur via the relatively slow process of nucleation and growth.

For a gas concentration of $30 \%$, Figure 6.1 clearly illustrates the formation of a bi-continuous network by spinodal decomposition (second snapshot at $t=0.66$ ). This breaks down in time to nucleate individual bubbles (third snapshot at $t=1.2$ ). We have run additional simulations to explore the nucleation stage at different gas concentrations. Figure 6.2 shows three simulations with initial gas concentration of $50 \%, 40 \%$ and $30 \%$. As before, the initial velocity is set to zero, and the uniform concentration is perturbed randomly in space by up to $\pm 5 \%$. In the symmetric case of $50 \%$ gas, a bi-continuous morphology appears and remains indefinitely. For the lower gas concentrations, on the other hand, the bi-continuous network breaks down to form gas bubbles. As the gas concentration is lowered, the phase separation becomes slower. It also takes longer for the simulation to reach an equilibrium state. We have also attempted a simulation with $15 \%$ gas, where the mixture is meta-stable. Nucleation and growth occurs only when we supply a large enough disturbance that drives the 

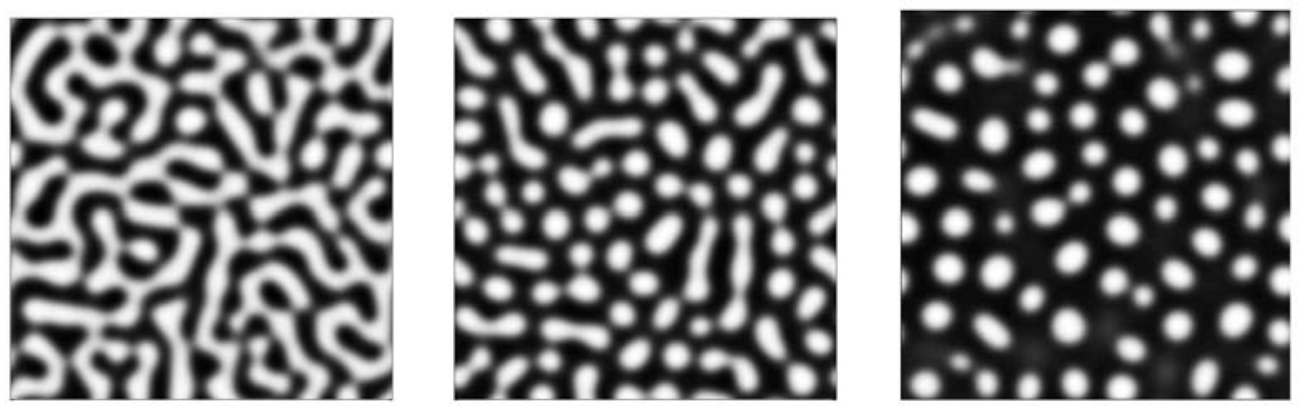

FIG. 6.2. Nucleation via spinodal decomposition with liquid/gas ratios of 50/50, 60/40, and 70/30. The snapshots are taken at times $t=0.5,1$, and 2, respectively.

local gas concentration about the threshold of $21.1 \%$. Otherwise the disturbance dies out.

In the context of foaming, "nucleation" refers to the appearance of a bubble-inmelt morphology that arises either from nucleation and growth or from the breakup of a bi-continuous network resulting from spinodal decomposition. Although the gas solubility is typically low for foaming materials, either process can be relevant depending on the pressure, temperature and other foaming conditions. In addition, nucleating agents are routinely added to generate spatial inhomogeneities in gas concentration and induce nucleation [15].

In the classical homogeneous nucleation theory, a key concept is the "critical bubble" that is in thermodynamic and mechanical equilibrium with its environment $[2,15,40]$. Local mass and momentum balances then determine the critical radius for the viability of the bubble. In our phase-field model, the Cahn-Hillard diffusion makes nucleation a nonlocal process. For example, the pressure inside an emerging bubble is not given by the local Laplace's law; rather it depends on the overall environment. This non-locality will be better appreciated when we discuss bubble-bubble interactions in the next sub-section.

6.2. Stage II: Bubble Growth at the Expense of Smaller Bubbles. After nucleation, the bubbles grow by diffusion of gas from the melt into the bubble. A salient feature of this process is Oswald ripening: the larger bubbles grow at the expense of the smaller ones [10]. To understand this process, we recognize that in this case the chemical potential is directly proportional to the mean curvature of the bubbles [11]. This can be derived directly through an asymptotic expansion of the chemical potential near the configuration. Thus, a larger bubble possesses a lower chemical potential and a smaller bubble a higher potential; the former will grow at 

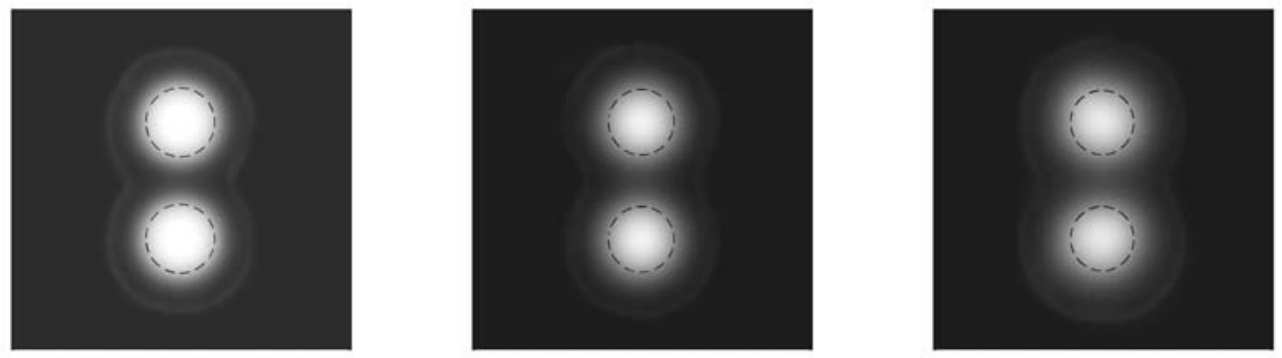

FIG. 6.3. Non-interaction between two small bubbles. The snapshots are at times $t=0,0.1125$ and 0.225 .
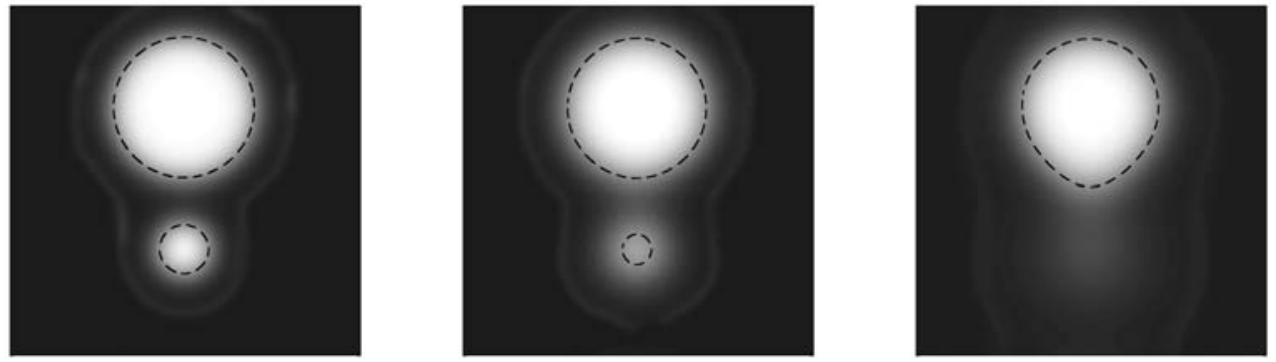

FIG. 6.4. Diffusion of smaller bubble into a larger one. The snapshots are at times $t=0,0.05$ and 0.1 .

the latter's expense. This phenomenon has been considered in classical models of foaming [40]. In that framework, it is explained by the fact that smaller bubbles have higher interior pressure (Laplace's law), which implies higher gas concentration at the surface (Henry's law) and thus reduced diffusion to the bubble.

To illustrate this mechanism, we carried out two additional simulations on bubblebubble interaction. The first has two small bubbles of equal size, radius 10, placed a distance of 14 apart (Figure 6.3). Since they possess equal chemical potential, the only interaction between them is the blurring of the region between them. There is no coalescence. In the second simulation, we place two bubbles of radius 20 and 7 a distance of 14 apart. Figure 6.4 shows that the smaller bubble diffuses into the larger. This is exactly the type of interaction that appears in the second stage of the foaming process $(t=1.5$ in Figure 6.1). In fact, one may consider this process as contributing to the coarsening of the bubbles; the boundary between stages II and III is therefore not a sharp one.

6.3. Stage III: Coarsening. When the growing bubbles get close enough to their neighbors, coalescence occurs, and the foam texture coarsens. This process 

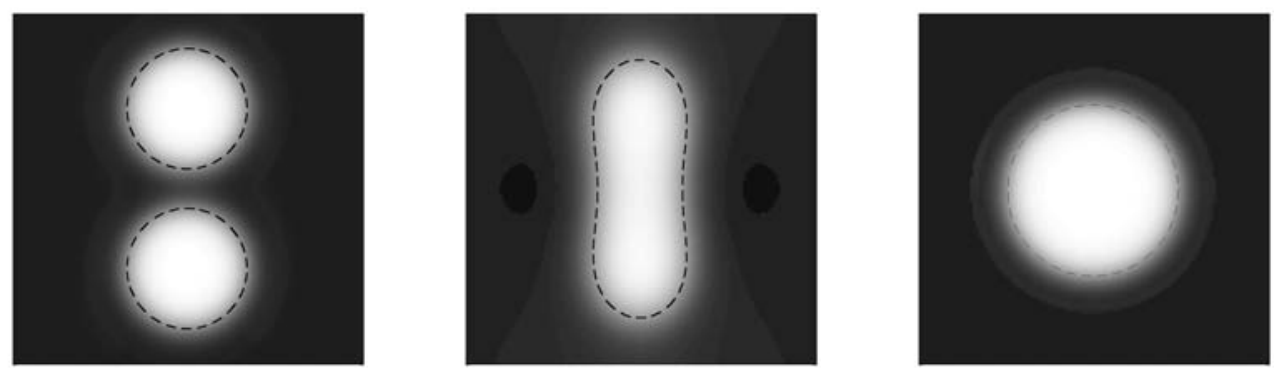

FIG. 6.5. Coarsening between two larger bubbles. The snapshots are at times $t=0,0.45$ and 1.35.

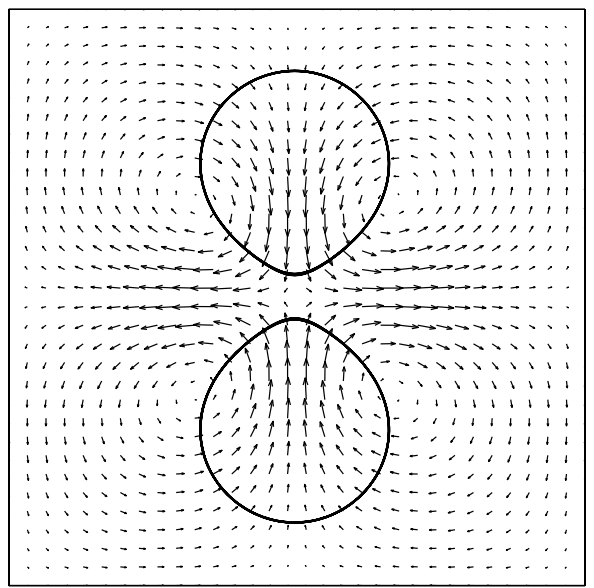

FIG. 6.6. Velocity field around the two coarsening bubbles at time $t=0.3$, showing drainage flow.

is evident in the last panel of Figure 6.1, and further illustrated by a coalescence simulation shown in Figure 6.5. In a square domain with side length 100, we place two bubbles, both of radius 17 , a distance of 11 between their respective $\phi=0$ boundaries. Soon after the simulation starts, the bubbles begin to deform and extend toward each other. Once they initially "touch", surface effects take over and they continue to deform until they have coalesced into a single large bubble.

This process differs from cell collapse in real foams in several aspects, and highlights the unconventional features of our diffuse-interface model. First, the coalescence during foaming is caused by the high pressure in neighboring bubbles squeezing the matrix fluid out of the film separating the bubbles. In Figure 6.5, the coalescence is driven by Cahn-Hillard diffusion. Figure 6.6 illustrates the flow field around the two merging bubbles. Although the local flow resembles the familiar film drainage picture [47], the underlying mechanism is not the same. Second, the final rupture of the film separating the two bubbles is believed to be mainly governed by short-range 
THE NUCLEATION AND GROWTH OF GAS BUBBLES IN A NEWTONIAN FLUID

forces such as van der Waals force. Since the Cahn-Hillard energy indeed represents inter-molecular forces, it gives rise to a similar short-range force, although the scaling behavior differs from that of the van der Waals force [50].

Finally, surfactants play major roles in real foams. Their effects can be incorporated into a phase-field model by including an energy functional that concentrates on the interfacial region. Details will be discussed in future publications.

7. Conclusion. This paper proposes a phase-field theory for gas nucleation and bubble dynamics in a liquid matrix with potential applications to polymer foaming. As compared with classical sharp-interface models, this theory has the advantage that various stages of foaming can be predicted naturally, based on a simple principle governing mixing and separation of fluids. In particular, nucleation and bubble coalescence, two processes that have largely eluded previous efforts, can now be fully incorporated. This avoids two difficulties in prior models: determination of initial conditions for bubble growth and tracking interfacial motions during bubble coalescence. We have established the relationship of the new model to classic models, illustrated its advantages by numerical simulations, and pointed out the limitations in its current form.

The energetic variational approaches guarantee that the resulting coupled systems are consistent with the thermodynamics - the basic energy laws. By relating the current approach to the classical empirical laws, with the advantage of easy incorporation of more complicated material properties, we conclude that this theory represents a promising new approach for complex interfacial problems as encountered in polymer foaming.

We conclude by noting the limitations of the current work in the context of a real foaming operation. First, the parameter values used here are chosen more for convenience than to mimic real materials. In particular, the density and viscosity of the two species differ greatly in reality. Though presenting no conceptual difficulties, incorporating these differences will entail a numerical effort. Second, foaming typically involves great variations in ambient pressure and temperature. These have not been considered. Third, the phase-field model implies transport laws that differ from the familiar Henry's law and Fick's law. To some extent, a connection can be made between the two approaches. But further study is needed to explore the implications of the differences to bubble growth in foaming. Finally, our study is limited to two dimensions. Currently we are developing general three-dimensional numerical codes that also allow unequal density and viscosity. To keep the problem size manageable in 
$3 \mathrm{D}$, we have developed moving-grid and adaptive meshing algorithms to better resolve the interfaces.

\section{REFERENCES}

[1] N. D. Alikakos, P. W. Bates, and X. F. Chen, Convergence of the Cahn-Hilliard equation to the Hele-Shaw model, Arch. Rational Mech. Anal., 128 (1994), pp. 165-205.

[2] A. Arefmanesh, S. G. Advani, and E. E. Michaelides, A numerical study of bubble growth during low pressure structural foam molding process, Polymer Eng. Sci., 30 (1990), pp. 1330-1337.

[3] G. K. Batchelor, An Introduction to Fluid Dynamics, Cambridge University Press, Cambridge, paperback ed., 1999.

[4] D. G. Bucknall, Influence of interfaces on thin polymer film behavior, Prog. Mater. Sci., 49 (2004), pp. 713-786.

[5] J. W. Cahn ANd J. E. Hillard, Free energy of a nonuniform system. I. Interfacial free energy, J. Chem. Phys., 28 (1958), pp. 258-267.

[6] Y. C. Chang, T. Y. Hou, B. Merriman, and S. Osher, A level set formulation of eulerian interface capturing methods for incompressible fluid flows, J. Comput. Phys., 124 (1996), pp. 449-464.

[7] X. Chen, J. J. Feng and C. A. Bertelo, Plasticization effects on bubble growth during polymer foaming, Polym. Eng. Sci. 46 (2006), pp. 97-107.

[8] I.-L. Chern, J. Glimm, O. McBryan, B. Plohr, and S. Yaniv, Front tracking for gas dynamics, J. Comput. Phys., 62 (1986), pp. 83-110.

[9] K. A. Cliffe And S. J. TAVener, Marangoni-Bénard convection with a deformable free surface, J. Comput. Phys., 145 (1998), pp. 193-227.

[10] P. G. DE Gennes, Dynamics of fluctuations and spinodal decomposition in polymer blends, J. Chem. Phys., 72 (1980), pp. 4756-4763.

[11] Q. Du, C. Liu, AND X. WAng, Simulating the deformation of vesicle membranes under elastic bending energy in three dimensions, J. Comput. Phys., 212 (2006), pp. 757-777.

[12] W. E And F. Oтto, Thermodynamically driven incompressible fluid mixtures, J. Chem. Phys., 107 (1998), pp. 10177-10184.

[13] W. E and P. Palffy-Muhoray, Phase separation in incompressible systems, Phys. Rev. E (3), 55 (1997), pp. R3844-R3846.

[14] D. A. Edwards, H. Brenner, And D. T. Wasan, Interfacial Transport Process and Rheology, Butterworth-Heinemann, 1991.

[15] J. J. Feng And C. A. Bertelo, Prediction of bubble growth and size distribution in polymer foaming based on a new heterogeneous nucleation model, J. Rheol., 48 (2004), pp. 439-462.

[16] J. J. Feng, C. Liu, J. Shen And P. Yue, An energetic variational formulation with phase field methods for interfacial dynamics of complex fluids: advantages and challenges, in Modeling of Soft Matter (IMA Volumes in Mathematics and its Applications, eds. M.-C. T. Calderer and E. Terentjev). pp. 1-26. Springer, New York (2005).

[17] W. M. Feng, P. Yu, S. Y. Hu, Z. K. Liu and L. Q. Chen, Spectral implementation of an adaptive moving mesh method for phase-field equations, J. Comput. Phys., 220 (2006), pp. $498-510$.

[18] F. Gibou, R. Fedkiw, R. Caflisch, and S. Osher, A level set approach for the numerical simulation of dendritic growth, J. Sci. Comput., 19 (2003), pp. 183-199.

[19] J. Glimm, J. W. Grove, X. L. Li, and D. C. Tan, Robust computational algorithms for dynamic interface tracking in three dimensions, SIAM J. Sci. Comput., 21 (2000), pp. 22402256 .

[20] J. Glimm, E. Isaacson, D. Marchesin, and O. McBryan, Front tracking for hyperbolic systems, Adv. in Appl. Math., 2 (1981), pp. 91-119.

[21] J. Glimm, X. L. Li, Y. Liu, AND N. ZhaO, Conservative front tracking and level set algorithms, Proc. Natl. Acad. Sci. USA, 98 (2001), pp. 14198-14201.

[22] M. E. Gurtin, An Introduction to Continuum Mechanics, vol. 158 of Mathematics in Science and Engineering, Academic Press, 1981.

[23] D. Klempner and K. C. Frisch, Handbook of Polymeric Foams and Foam Technology, Hanser, 1991.

[24] V. V. Krotov and A. I. Rusanov, Physicochemical Hydrodynamics of Capillary Systems, Inperial College Press, London, 1999.

[25] L. D. Landau and E. M. Lifshitz, Fluid Mechanics, Pergamon Press, 2 ed., 1987. 
[26] L. D. Landau and E. M. Lifshitz, Statistical Physics, Butterworth-Heinemann, 1996.

[27] A. M. Lapena, S. C. Glotzer, S. A. Langer, and A. J. Liu, Effect of ordering on spinodal decomposition of liquid-crystal/polymer mixtures, Phy. Rev. E, 60 (1999), pp. R29-R32.

[28] R. G. LARson, The Structure and Rheology of Complex Fluids, Oxford, 1995.

[29] J. Li And Y. Renardy, Numerical study of flows of two immiscible liquids at low Reynolds number, SIAM Review, 42 (2000), pp. 417-439.

[30] - Shear-induced rupturing of a viscous drop in a Bingham liquid, J. Non-Newtonian Fluid Mech., 95 (2000), pp. 235-251.

[31] C. Liu And J. Shen, A phase field model for the mixture of two incompressible fluids and its approximation by a fourier-spectral method, Physica D, 179 (2003), pp. 211-228.

[32] C. Liu, J. Shen, J. J. Feng, And P. Yue, Variational approach in two-phase flows of complex fluids: transport and induced elastic stress, in Mathematical Models and Methods in Phase Transitions (ed. by A. Miranville). Chapter 11, pp. 259-278. Nova Publications, New York (2004).

[33] C. Liu, S. J. Tavener, and N. J. Walkington, A variational phase field model for MarangoniBénard convection with a deformable free surface, Preprint, (2001).

[34] C. Liu and N. J. Walkington, Approximation of liquid crystal flows, SIAM Journal on Numerical Analysis, 37 (2000), pp. 725-741.

[35] — An eulerian description of fluids containing visco-hyperelastic particles, Arch. Rat. Mech. Ana., 159 (2001), pp. 229-252.

[36] B. Merriman, J. Bence, And S. Osher, Motion of multiple junctions, a level set approach, Journal of Computational Physics, 112 (1994), pp. 334-363.

[37] S. Osher and R. Fedkiw, The Level Set Method and Dynamic Implicit Surfaces, SpringerVerlag, New York, NY, 2002.

[38] S. Osher And J. Sethian, Fronts propagating with curvature dependent speed: Algorithms based on Hamilton Jacobi formulations, Journal of Computational Physics, 79 (1988), pp. $12-49$.

[39] R. F. Probstein, Physicochemical Hydrodynamics, An Introduction, John Wiley and Sons, INC., 1994.

[40] N. S. Ramesh, D. H. Rasmussen, and G. A. Campbell, The heterogeneous nucleation of microcellular foams assisted by the survival of microvoids in polymers containing low glass transition particles. part $i$ : mathematical modeling and numerical simulation, Polym. Eng. Sci., 34 (1994), pp. 1685-1696.

[41] A. D. ReY, Viscoelastic theory for nematic interfaces, Physical Review E, 61 (2000), pp. 15401549.

[42] S. Roy, W. Q. Xu, S. J. Park, And K. M. Liechti, Anomalous diffusion of a penetrant in a viscoelastic polymer: Modelling and testing, Polym. Polym. Compost., 8 (2000), pp. 295305.

[43] M. Sanopoulou, D. F. Stamatialis, and J. H. Petropoulos, Investigation of case II diffusion behavior. 1. Theoretical studies based on the relaxation dependent solubility model, Macromolecules, 35 (2002), pp. 1012-1020.

[44] J. Sandler, F. Wollecke, V. Altstadt, E. Wettstein, and D. Rakutt, Principal correlation of pvc melt elongational properties with foam cell morphology, Cellular Polym., 19 (2000), pp. 371-388.

[45] M. A. Shafi and R. W. Flumerfelt, Initial bubble growth in polymer foam processes, Chem. Eng. Sci., 52 (1997), pp. 627-633.

[46] D. C. Venerus And N. YAla, Transport analysis of diffusion-induced bubble growth and collapse in viscous liquids, AIChE J., 43 (1997), p. 2948-2959.

[47] H. Yang, C. C. Park, Y. T. Hu, L. G. Leal, The coalescence of two equal-sized drops in a two-dimensional linear flow, Phys. Fluids, 13 (2004), pp. 1087-1106.

[48] X. Yang, J. J. Feng, C. Liu And J. Shen, Contraction and pinch-off phenomena of a liquid filament, J. Comput. Phys., 218 (2006), pp. 417-428.

[49] P. Yue, J. Feng, C. Liu, And J. Shen, A diffuse-interface method for simulating two-phase flows of complex fluids, J. Fluid Mech., 515 (2004), pp. 293-317.

[50] P. Yue, J. J. Feng, C. Liu, And J. Shen, Diffuse-interface simulations of drop coalescence and retraction in viscoelastic fluids, J. Non-Newtonian Fluid Mech., 129 (2005), pp. 163-176.

[51] P. Yue, J. J. Feng, C. Liu, And J. Shen, Transient drop deformation upon startup of shear in viscoelastic fluids, Phys. Fluids, 17 (2005), Article no. 123101.

[52] P. Yue, J. J. Feng, C. Liu, and J. Shen, Viscoelastic effects on drop deformation in steady shear, J. Fluid Mech., 540 (2005), pp. 427-437.

[53] P. Yue, J. J. Feng, C. Liu, And J. Shen, Interfacial forces and Marangoni flow on a nematic drop retracting in an isotropic fluid, J. Colloid Interface Sci., 290 (2005), pp. 281-288. 
[54] P. Yue, C. Zhou, J. J. Feng, C. F. Ollivier-Gooch and H. H. Hu, Phase-field simulations of interfacial dynamics in viscoelastic fluids using finite elements with adaptive meshing, J. Comput. Phys., 219 (2006), pp. 47-67.

Department of Mathematics, Princeton University, Princeton, NJ 08544 (aaronnaber@usa.com).

Department of Mathematics, Pennsylvania State University, University Park, PA 16802 (liu@math.psu.edu).

Department of Chemical and Biological Engineering and Department of Mathematics, University of British Columbia, Vancouver, BC V6T 1Z4, Canada (jfeng@chml.ubc.ca). 